POSTER

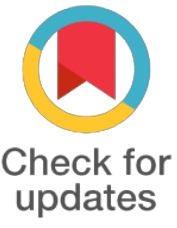

${ }^{*}$ For correspondence:

dtlong@hcmus.edu.vn

Competing interests: The authors declare that no competing interests exist.

Received: 2017-02-07

Accepted: 2017-08-19

Published: 2017-09-05

Copyright The Author(s) 2017. This article is published with open access by BioMedPress (BMP).

This article is distributed under the terms of the Creative Commons Attribution License (CC-BY 4.0) which permits any use, distribution, and reproduction in any medium, provided the original author(s) and the source are credited.

\section{Experimental culturing of chick embryo in shell-less culture system - the first research in Vietnam}

\author{
Quy Xuan Nguyen, Long Thanh Dang \\ Laboratory of Stem Cell Research and Application, University of Science, Vietnam National \\ University HCMC, Ho Chi Minh City, Vietnam
}

\section{Abstract}

Chick embryo is an ideal model with numerous applications in biomedical research. Among a variety of methods have been carried out for culturing chick embryos, shell-less culture system has a large number of advantages on accessibility, observation and manipulation. In this study, chick embryos were transferred to the shell-less culture system and the development of the chick embryos were assessed. Correlation between the diameter of sinus terminalis on the surface of yolk sac and viability of the embryos would be evaluated. In addition, calcium lactate was added to the culture system in order to find out the optimal amount. After the experiments, results showed that there was no difference between embryos in shell-less culture system and traditional method during incubation period. Secondly, the proportion of live chick embryos until embryonic day 17 reached the highest rate at $87,5 \%$ when the diameter of sinus terminalis was between 16 and $21 \mathrm{~mm}$. At last, there was no significant difference between the group with $250 \mathrm{mg}$ calcium lactate supplemented as compared to no supplemented group. Calcium lactate had a lethal effect on chick embryos when the supplemented content was $550 \mathrm{mg}$. In conclusion, the shell-less culture system could be able to allow the survival of chick embryos until day 21 , with high rate in day 17. Besides, this has been the very first time the shell-less culture system was performed in Vietnam.

\section{Keywords}

Calcium lactate, chick embryo, shell-less culture system, sinus terminalis.

Funding

University of Science with T2017-45 project.

\section{References}

